Erratum

Nicolás Andruskiewitsch(D) Giovanna Carnovale(D) Gastón Andrés García(iD

\title{
Erratum to: Finite-dimensional pointed Hopf algebras over finite simple groups of Lie type V. Mixed classes in Chevalley and Steinberg groups
}

Published online: 16 October 2020

Erratum to: manuscripta math. https://doi.org/10.1007/s00229-020-01248-5

The article title was originally published with typographical error. This has been corrected through this erratum. Original article has been corrected.

Publisher's Note Springer Nature remains neutral with regard to jurisdictional claims in published maps and institutional affiliations.

The original article can be found online at https://doi.org/10.1007/s00229-020-01248-5. N. Andruskiewitsch: CIEM - CONICET, FaMAF, Universidad Nacional de Córdoba, Medina Allende s/n, Ciudad Universitaria, 5000 Córdoba, Argentina e-mail: andrus@famaf.unc.edu.ar URL: https://www.famaf.unc.edu.ar/ andrus/

G. Carnovale $(\varangle)$ : Dipartimento di Matematica Tullio Levi-Civita, Università degli Studi di Padova, via Trieste 63, 35121 Padua, Italy e-mail: carnoval@math.unipd.it

G. A. García: CONICET, Departamento de Matemática, Facultad de Ciencias Exactas, Universidad Nacional de La Plata, C. C. 172, 1900 La Plata, Argentina e-mail: ggarcia@mate.unlp.edu.ar

URL: http://www.mate.unlp.edu.ar/ ggarcia/ 